\title{
A parallel algorithm for the enumeration of self-avoiding polygons on the square lattice
}

\author{
Iwan Jensen \\ Department of Mathematics \& Statistics, The University of Melbourne \\ Vic. 3010, Australia
}

November 7, 2018

\begin{abstract}
We have developed a parallel algorithm that allows us to enumerate the number of self-avoiding polygons on the square lattice to perimeter length 110 . We have also extended the series for the first 10 area-weighted moments and the radius of gyration to 100. Analysis of the resulting series yields very accurate estimates of the connective constant $\mu=2.63815853031(3)$ (biased) and the critical exponent $\alpha=0.5000001(2)$ (unbiased). In addition we obtain very accurate estimates for the leading amplitudes confirming to a high degree of accuracy various predictions for universal amplitude combinations.
\end{abstract}

\section{Introduction}

A self-avoiding polygon (SAP) on a lattice can be defined as a walk along the edges of the lattice which starts and ends at the origin but has no other self-intersections. Alternatively we can define a SAP as a graph whose vertices are of degree 0 or 2 and apart for isolated vertices has only a single component. The enumeration of self-avoiding polygons on various lattices is an interesting combinatorial problem in its own right, and is also of considerable importance in the statistical mechanics of lattice models [15]. When enumerated by perimeter SAPs can be considered a model for ring polymers and when enumerated by area they model vesicles [21, 10, 11].

The basic problems are the calculation of the number $p_{n}$ of polygons of perimeter $n$, the number $a_{m}$ of polygons of area $m$, or more generally the number $p_{m, n}$ of polygons of area $m$ and perimeter $n$. Note that on the square lattice polygons have an even perimeter and $p_{n}=0$ for $n$ odd. Here we are interested in area-weighted moments, where the $k^{\prime}$ 'th area-weighted moment is $\left\langle a^{k}\right\rangle_{n}=\left(\sum_{m} m^{k} p_{m, n}\right) / p_{n}$. Also of great interest is the mean-square radius of gyration $\left\langle R^{2}\right\rangle_{n}$, which measures the typical size of a polygon with perimeter $n$. These quantities are expected to behave as

$$
\begin{aligned}
p_{n} & =B \mu^{n} n^{\alpha-3}[1+o(1)], \\
\left\langle a^{k}\right\rangle_{n} & =E^{(k)} n^{2 k \nu}[1+o(1)], \\
\left\langle R^{2}\right\rangle_{n} & =D n^{2 \nu}[1+o(1)],
\end{aligned}
$$


where $\mu$ is the so-called connective constant, while $\alpha$ and $\nu$ are critical exponents. When analyzing the data it is often convenient to use the associated generating functions

$$
\begin{aligned}
\mathcal{R}_{g}^{2}(u) & =\sum_{n} n^{2} p_{n}\left\langle R^{2}\right\rangle_{n} u^{n}=\sum_{n} r_{n} u^{n} \sim R(u)(1-u \mu)^{-(\alpha+2 \nu)}, \\
\mathcal{P}^{(k)}(u) & =\sum_{n} p_{n}\left\langle a^{k}\right\rangle_{n} u^{n}=\sum_{n} a_{n}^{(k)} u^{n} \sim A^{(k)}(u)(1-u \mu)^{2-(\alpha+2 k \nu)} .
\end{aligned}
$$

where the various factors are chosen so that $r_{n}$ and $a_{n}^{(k)}$ are integers. These series are thus expected to have a singularity at the critical point $u_{c}=1 / \mu$ with critical exponents as above. In particular we note that the critical exponent of the perimeter generating function, $\mathcal{P}(u)=\mathcal{P}^{(0)}(u)$, is $2-\alpha$.

Despite strenuous effort over the past 50 years or so this problem has not been solved on any regular two dimensional lattice. However, for the hexagonal lattice the critical point, $u_{c}^{2}=1 /(2+\sqrt{2})$ as well as the critical exponents $\alpha=1 / 2$ and $\nu=3 / 4$ are known exactly [25], though non-rigorously. Very firm evidence exists from previous numerical work that the exponent $\alpha$ is universal and thus equals $1 / 2$ for all two dimensional lattices [13, 9, 17, 24]. The value of $\nu$ and its universality have also been confirmed by numerical work [26, 13, 8, 24, 16.

It is also known [2] that the amplitude combination $E^{(1)} / D$ is universal, and that

$$
B D=\frac{5}{32 \pi^{2}} \sigma a_{0}
$$

where $a_{0}$ is the area per site and $\sigma$ is an integer such that $p_{n}$ is non-zero only if $n$ is divisible by $\sigma$. For the square lattice $a_{0}=1$ and $\sigma=2$. These predictions have been confirmed numerically [2, 23, 24, 16, 22].

Recently, Richard et al. [27] found, subject to a very reasonable conjecture, the exact scaling function for self-avoiding polygons. This in turn led to the derivation of universal amplitude combinations for all the $E^{(k)}$, namely that $E^{(k)} B^{k-1}$ are known universal constants. In particular it has been shown that $E^{(1)}=1 / 4 \pi$ [1]. These predictions were strongly supported by numerical evidence [27.

Some years ago [3] it was pointed out that since the hexagonal lattice connective constant is given by the zero of a quadratic in $u^{2}$, it is plausible that this might be the case also for the square lattice connective constant. It was found that $581 u^{4}+7 u^{2}-13$ was the only polynomial with "small" integer coefficients consistent with this estimate. The relevant zero of this polynomial is $u_{c}^{2}=0.1436806292698685 \ldots$. In [18] the numerical evidence was in complete agreement with this conjecture, but with 4 more significant digits than when the original suggestion was made.

This paper builds on the work of Enting [6] who enumerated square lattice polygons to 38 steps using the finite lattice method. Using the same technique this enumeration was extended by Enting and Guttmann to 46 steps [7] and later to 56 steps [13] and further extended to 70 steps in unpublished work. These extensions to the enumeration were largely made possible by improved computer technology. Jensen and Guttmann [18] improved the algorithm and extended the enumeration to 90 steps while using essentially the same computational resources used to obtain polygons to 70 steps. The work by Guttmann and Enting [13] also included calculations of moments of the caliper size distribution. Hiley and Sykes [14] obtained the number of square lattice polygons by both 
area and perimeter up to perimeter 18. Enting and Guttmann extended the calculation to perimeter 42 [8]. The radius of gyration was calculated for SAPs up to 28 steps by Privman and Rudnick [26]. Jensen [16] extended the series for area-moments with $k \leq 2$ and the radius of gyration to 82 steps. In [27] the calculation for area-moments was extended to $k \leq 10$.

The main purpose of this paper is to report on a new parallel version of our earlier algorithms which allows us to significantly extend the series and use these extended series to critically examine the theoretical predictions given above as well as revisit the conjecture for the connective constant on the square lattice. Using the parallelised algorithm and a new superior memory management, inspired by Knuth's work on the enumeration of polyominoes [20], we have been able to extend the enumeration of square lattice polygons to 110 steps. We extend the series for area-weighted moments with $k \leq 10$ and the radius of gyration to 100 steps.

In the next section we will very briefly review the finite lattice method for enumerating square lattice polygons and give some details of the improved parallel algorithm. The results of the analysis of the series are presented in Section 3 including a detailed discussion of the conjecture for the exact critical point and numerical tests of the predictions for universal amplitude combinations.

\section{Enumeration of polygons}

The algorithm used to enumerate SAPs on the square lattice is an enhancement of the finite-lattice method devised by Enting [6] in his pioneering work, which contains a detailed description of the original approach. A major enhancement, resulting in an exponentially more efficient algorithm, is described in some detail in [18] while details of the changes required to enumerate area-moments and the radius of gyration can be found in [16. In the following we shall briefly outline those parts of the method required to understand how the parallel version works.

\subsection{The transfer matrix algorithm}

The first terms in the series for the polygon generating function can be calculated using transfer matrix techniques to count the number of polygons in rectangles $W$ vertices wide and $L$ vertices long. Due to the symmetry of the square lattice one need only consider rectangles with $L \geq W$. In the original application [6] valid polygons were required to span the enclosing rectangle in the lengthwise direction. Clearly polygons which are narrower than the width of the rectangle are counted many times. It is, however, easy to obtain the polygons of width exactly $W$ and length exactly $L$ from this enumeration [6]. Any polygon spanning such a rectangle has a perimeter of length at least $2(W+L)-4$. By adding the contributions from all rectangles of width $W \leq W_{\max }$ (where the choice of $W_{\max }$ depends on available computational resources) and length $W \leq L \leq 2 W_{\max }-W+1$, with contributions from rectangles with $L>W$ counted twice, the number of polygons per vertex of an infinite lattice is obtained correctly up to perimeter $N_{\max }=4 W_{\max }-2$.

The transfer matrix technique involves drawing a boundary line through the rectangle intersecting a set of up to $W+1$ edges. Polygons in a given rectangle are enumerated by moving the boundary line so as to add one vertex at a time, as shown in Fig. 1. 


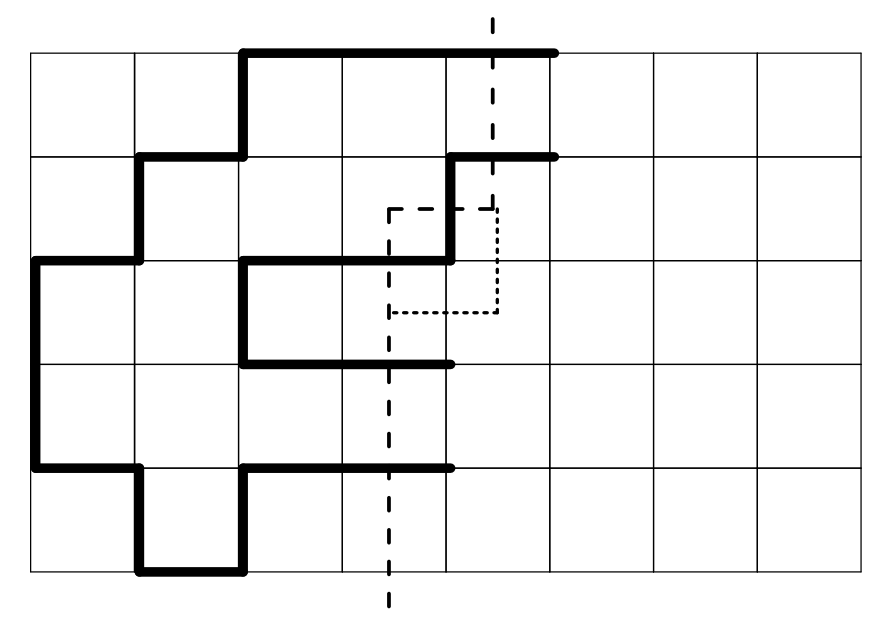

Figure 1: A snapshot of the boundary line (dashed line) during the transfer matrix calculation on the square lattice. Polygons are enumerated by successive moves of the kink in the boundary line, as exemplified by the position given by the dotted line, so that one vertex at a time is added to the rectangle. To the left of the boundary line we have drawn an example of a partially completed polygon.

In this fashion we build up the rectangle column by column with each column built up vertex by vertex. As we move the boundary line it intersects partially completed polygons consisting of disjoint loops that must all be connected to form a single polygon. For each configuration of occupied or empty edges along the intersection we maintain a (perimeter) generating function for open loops to the left of the line cutting the intersection in that particular pattern. The updating of the generating functions depends primarily on the configuration of the two edges at the kink in the boundary line prior to the move (we shall refer to these edges as the kink edges). As the boundary line is moved the two new edges intersected by the boundary line can be either empty or occupied.

To avoid situations leading to graphs with more than a single component we have to forbid a loop to close on itself if the boundary line intersects any other loops. So two loop ends can only be joined if they belong to different loops or all other edges are empty. To exclude loops which close on themselves we need to label the occupied edges in such a way that we can easily determine whether or not two loop ends belong to the same loop. The most obvious choice would be to give each loop a unique label. However, on two-dimensional lattices there is a more compact scheme relying on the fact that two loops can never intertwine. Each end of a loop is assigned one of two labels depending on whether it is the lower end or the upper end of a loop. Each configuration along the boundary line can thus be represented by a set of edge states $\left\{\sigma_{i}\right\}$, where

$$
\sigma_{i}= \begin{cases}0 & \text { empty edge } \\ 1 & \text { lower end of a loop } \\ 2 & \text { upper end of a loop. }\end{cases}
$$

Configurations are read from the bottom to the top. The configuration along the intersection of the partially completed polygon in Fig. 1 is $\{011 \overline{21} 22\}$ before the move, where we use over-lining to indicate the kink edges, and $\{01 \overline{10} 022\}$ after the move. It is easy to see that this encoding uniquely describes which loop-ends are connected. In order to find the 

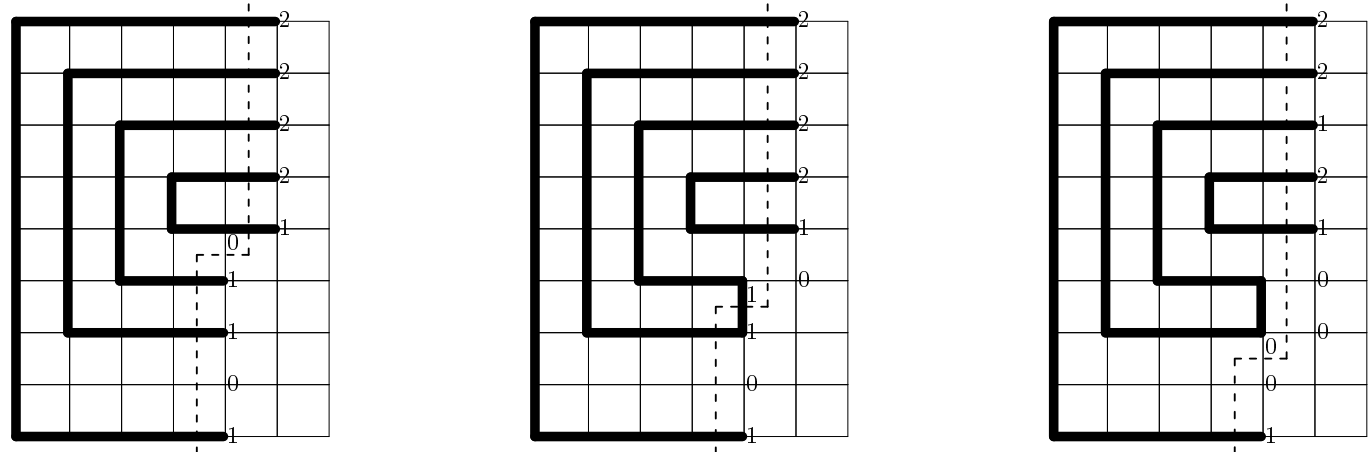

Figure 2: Snapshots of the boundary line (dashed line) during the TM calculation. Shown is a situation with four nested loops (left panel) where the lower ends of two loops are joined (middle panel) resulting in a situation with three nested loops (right panel) and a relabeling of the loop ends.

upper loop-end, matching a given lower end, we start at the lower end and work upwards in the configuration counting the number of ' 1 's and ' 2 's we pass (the ' 1 ' of the initial lower end is not included in the count). We stop when the number of ' 2 's exceeds the number of ' 1 's. This '2' marks the matching upper end of the loop. Ignoring the '0's the '1's and '2's can be viewed as perfectly balanced parenthesis. Those familiar with algebraic languages will recognize that each configuration of labeled loop-ends forms a Motzkin word [5]. It is known that the number of Motzkin words of length $m$ grows exponentially like $3^{m}$. This means that the number of configurations and thus the computational complexity of the FLM calculation grows like $3^{N_{\max } / 4}$.

The rules for updating the partial generating functions as the intersection is moved are identical to the original work, so we refer the interested reader to [6] for further details regarding this aspect of the transfer matrix calculation. The only important aspect we wish to emphasize here is that when joining two loop-ends at the kink we may have to change the labeling of a corresponding loop-end elsewhere in the resulting new configuration. An example is shown in Fig. 2. In this case we start out with four nested loops corresponding to the configuration $\{101 \overline{10} 12222\}$, then upon moving the kink in the boundary line the lower loop-ends of the second and third loops are joined leading to the configuration $\{10 \overline{11} 012222\}$. After the next move we see that there are now three differently nested loops and the upper end of the second loop (prior to the moves) have become the lower end of the third loop (after the moves) resulting in the final configuration $\{1 \overline{00} 0012122\}$.

The major improvement to the original method as explained in [18] is that we require valid polygons to span the rectangle in both directions. In other words we directly enumerate polygons of width exactly $W$ and length $L$ rather than polygons of width $\leq W$ and length $L$ as was done originally. At first glance this would appear to be inefficient since for many boundary line configurations we now have to keep 4 distinct generating functions depending on which borders have been touched. However, as demonstrated in practice [18] it actually leads to an algorithm which is both exponentially faster and whose memory requirement is exponentially smaller. Experimentally it was found that the computational complexity was close to $2^{N_{\max } / 4}$, much better than the $3^{N_{\max } / 4}$ of the original approach. Realizing the full savings in time and memory usage require enhancements to 
the original algorithm. The most important is what we call pruning. This procedure, details of which are given in 18, allows us to discard most of the possible configurations for large $W$ because they only contribute to polygons of length greater than $N_{\max }$. Briefly this works as follows. Firstly, for each configuration we keep track of the current minimum number of steps $N_{\text {cur }}$ already inserted to the left of the boundary line in order to build up that particular configuration. Secondly, we calculate the minimum number of additional steps $N_{\text {add }}$ required to produce a valid polygon. There are three contributions, namely the number of steps required to close the polygon, the number of steps needed (if any) to ensure that the polygon touches both the lower and upper border, and finally the number of steps needed (if any) to extend at least $W$ edges in the length-wise direction (remember we only need rectangles with $L \geq W$ ). If the sum $N_{\text {cur }}+N_{\text {add }}>N_{\text {max }}$ we can discard the partial generating function for that configuration, and of course the configuration itself, because it won't make a contribution to the polygon count up to the perimeter lengths we are trying to obtain.

Inspired by Knuth's algorithm for the enumeration of polyominoes [20], we implemented a couple of further enhancements to our SAP algorithm. The first improvement is a superior memory management. A given boundary line configuration does not contribute until order $N=N_{\text {cur }}+N_{\text {add }}$, so we need only retain the first $\left(N_{\text {max }}-N\right) / 2$ terms in the associated generating function, the factor of 2 arising since every other term is identically 0 . In our case the maximum in memory consumption occur at $W=24$ where there are approximately $8.1 \times 10^{8}$ distinct configurations and a total of about $2.1 \times 10^{9}$ non-zero terms in the generating functions. So on average there is only about 2.5 nonzero terms per configuration. The second improvement uses a further symmetry of the square lattice. When a column has been completed the configuration are symmetric under reflection. That is the generating functions for the configurations such as, $\{010122000\}$ and $\{000112020\}$, are identical. This symmetry also extends to the touching of the upper/lower borders of the rectangle.

The generalization of the algorithm to calculations of area-weighted moments and the radius of gyration is described in [16]. Note that the additional symmetry mentioned above does not extend to the radius of gyration calculation.

\subsection{Parallelization}

The computational complexity of the FLM grows exponentially with the number of terms one wishes to calculate. It is therefore little wonder that implementations of the algorithms have always been geared towards using the most powerful computers available. In the past decade or so parallel computing has become the paradigm for high performance computing. The early machines were largely dedicated MPP machines which more recently have been super-seeded by clusters.

The transfer-matrix algorithms used in the calculations of the finite lattice contributions are eminently suited for parallel computations.

The most basic concerns in any efficient parallel algorithm is to minimise the communication between processors and ensure that each processor does the same amount of work and use the same amount of memory. In practice one naturally has to strike some compromise and accept a certain degree of variation across the processors.

One of the main ways of achieving a good parallel algorithm using data decomposition is to try to find an invariant under the operation of the updating rules. That is we 
seek to find some property about the configurations along the boundary line which does not alter in a single iteration. The algorithm for the enumeration of polygons is quite complicated since not all possible configurations occur due to pruning and an update at a given set of edges might change the state of an edge far removed, e.g., when two lower loop-ends are joined we have to relabel one of the associated upper loop-ends as a lower loop-end in the new configuration (see Fig. 2). However, there still is an invariant since any edge not directly involved in the update cannot change from being empty to being occupied and vice versa. That is only the kink edges can change their occupation status. This invariant allows us to parallelise the algorithm in such a way that we can do the calculation completely independently on each processor with just two redistributions of the data set each time an extra column is added to the lattice.

The main points of the algorithm are summarized below:

1. With the boundary line in an upright position distribute the data across processors so that configurations with the same occupation pattern along the lower half of the boundary line are placed on the same processor.

2. Do the TM update inserting the top-half of a new column. This can be done independently by each processor because the occupation pattern in the lower half remains unchanged.

3. Upon reaching the half-way mark redistribute the data so that configurations with the the same occupation pattern along the upper half of the boundary line are placed on the same processor.

4. Do the TM update inserting the bottom-half of a new column.

5. Go back to 1 .

The redistribution among processors is done as follows:

1. On each processor run through the configurations to establish the configuration pattern $c$ of each configuration and calculate, $n(c)$, the number of configurations with a given pattern.

2. Calculate the sum of $n(c)$ on say processor 0 .

3. Sort $n(c)$ on processor 0 .

4. On processor 0 assign each pattern to a processor $p(c)$ such that:

(a) Set $p_{i d}=0$.

(b) Assign the most frequent unassigned pattern $c$ to processor $p_{i d}$.

(c) If the number of configurations assigned to $p_{i d}$ is less than the number of configurations assigned to $p_{0}$ then assign the least frequent unassigned patterns to $p_{i d}$ until the desired inequality is achieved.

(d) set $p_{i d}=p_{i d} \bmod N_{p}$, where $N_{p}$ is the number of processors.

(e) Repeat from (b) until all patterns have been assigned. 
Table 1: CPU-time and memory use for the parallel algorithm for enumerating polygons of maximal perimeter 98 at width 22 .

\begin{tabular}{rrrrrrr}
\hline Proc. & CPU time & Elapsed time & Max Conf & Min Conf & Max Term & Min Term \\
\hline 1 & $33: 26$ & $33: 34: 30$ & 94858092 & & 202124719 & \\
2 & $34: 58$ & $17: 31: 09$ & 45332715 & 45312242 & 99729074 & 99050619 \\
4 & $34: 15$ & $8: 35: 57$ & 22762665 & 22667218 & 51880015 & 51263646 \\
8 & $34: 03$ & $4: 16: 51$ & 11692292 & 11525456 & 26498730 & 26097260 \\
16 & $34: 16$ & $2: 09: 40$ & 5880705 & 5707628 & 13523912 & 13037482 \\
32 & $33: 15$ & $1: 03: 04$ & 2941787 & 2821055 & 6934653 & 6451282 \\
64 & $32: 29$ & $31: 07$ & 1489116 & 1398768 & 3519013 & 3222199 \\
\hline
\end{tabular}

5. Send $p(c)$ to all processors.

6. On each processor run through the configurations sending each configuration to its assigned processor.

The bulk of the calculations were performed on the facilities of the Australian Partnership for Advanced Computing (APAC). The APAC facility is a Compaq Server Cluster with 125 ES45's each with 41 Ghz chips for a total of 500 processors in the compute partition. The cluster has a total peak speed over 1Tflop. Each server node has at least 4 Gb of memory. Nodes are interconnected by a fat-tree low latency (MPI $<5$ usecs), high bandwidth (250 Mb/sec bidirectional) Quadrics network.

In Table 1 we have listed the time and memory use of the algorithm for $N_{\max }=98$ at $W=22$ using from 1 to 64 processors. The memory use of the single processor job was about $3 \mathrm{~Gb}$. As can be seen the algorithm scales perfectly from 1 to 64 processors since the total CPU time (column 2) stays almost constant while the elapsed time is halved when the number of processors is doubled. We expect that the rather surprising drop in CPU time at 32 or 64 processors is caused by better single processor optimization by the compiler. One would for example expect that the average time taken to fetch elements from main memory drops as the memory size on each individual processor drops from $3 \mathrm{~Gb}$ for the computation using a single processor to just under $50 \mathrm{Mb}$ for the 64 processor computation. Another main issue in parallel computing is that of load balancing, that is, we wish to ensure to the greatest extent possible that the workload is shared equally among all the processors. As can be seen this algorithm is quite well balanced. Even with 64 processors, where each processor uses only about 50Mb of memory, the difference between the processor handling the maximal and minimal number of configurations is less than $10 \%$. The same holds true for the total number of terms retained in the generating functions.

A simple timing of the various sub-routines of the parallel algorithm shows that the typical time to do a redistribution is the same as the average time taken per iteration in order to move the kink once. Since the maximal time use at $N_{\max }=110$ occurs at $W=24$ there are 24 iteration and just 2 redistributions per added column, so the overall cost of parallel execution is smaller than $10 \%$. 
Table 2: The number, $p_{n}$, of embeddings of $n$-step polygons on the square lattice. Only non-zero terms are listed.

\begin{tabular}{rrrr}
\hline \hline$n$ & $p_{n}$ & $n$ & $p_{n}$ \\
\hline 92 & 3959306049439766117380237943449096 & 102 & 49985425311177130573540712929060556804 \\
94 & 26117050944268596220897591868398452 & 104 & 331440783010043009106782321492277936522 \\
96 & 172472018113289556124895798382016316 & 106 & 2199725502650970871182263620080571090156 \\
98 & 1140203722938033441542255979068861816 & 108 & 14612216410979678692651320184958285074180 \\
100 & 7545649677448506970646886033356862162 & 110 & 97148177367657853074723038687712338567772 \\
\hline \hline
\end{tabular}

\subsection{Further details}

Finally a few remarks of a more technical nature. The number of contributing configurations becomes very sparse in the total set of possible states along the boundary line and as is standard in such cases one uses a hash-addressing scheme. Since the integer coefficients occurring in the series expansion become very large, the calculation was performed using modular arithmetic [19]. This involves performing the calculation modulo various integers $p_{i}$ and then reconstructing the full integer coefficients at the end. The $p_{i}$ are called moduli and must be chosen so they are mutually prime, e.g., none of the $p_{i}$ have a common divisor. The Chinese remainder theorem ensures that any integer has a unique representation in terms of residues. If the largest absolute values occurring in the final expansion is $m$, then we have to use a number of moduli $k$ such that $p_{1} p_{2} \cdots p_{k} / 2>m$. Since we are using a heavily loaded shared facility CPU time was more of a immediate limitation than memory and secondly more memory was used for the date required to specify the configuration and manage the storage than for storing the actual terms of the generating functions. So we used the moduli $p_{0}=2^{62}, p_{1}=2^{62}-1$ and $p_{2}=2^{62}-3$, which allowed us to represent $p_{n}$ correctly using these three moduli. The calculation of the area-weighted moments and the radius of gyration require a lot more memory for the generating functions (plus the radius of gyration calculation involves multiplication with quite large integers) so in this case we used prime numbers of the form $2^{30}-r_{i}$ for the moduli $p_{i}$. Up to 6 primes were needed to represent the coefficients correctly.

Combining all the memory minimization tricks mentioned above allows us to extend the series for the square lattice polygon generating function from 90 terms to 110 terms using at most $36 \mathrm{~Gb}$ of memory. The calculations requiring the most resource were at widths 23-25. These cases were done using 40 processors and took about 8-10 hours each per prime. The total CPU time required was about 1500 hours per prime. Obviously the calculation for each width and prime are totally independent and several calculations can be done simultaneously. A similar total amount of resources was required to calculate the area-moments and the radius of gyration.

In Table 2 we have listed the new terms obtained in this work for the number of polygons with perimeter $92-110$. The number of polygons of length $\leq 56$ can be found in [13. while those up to length 90 are listed in [18].

\section{Analysis of the series}

To obtain the singularity structure of the generating functions we used the numerical method of differential approximants [12. Since all odd terms in the series are zero and 
the first non-zero term is $p_{4}$ we actually analyzed the function $P(u)=\sum_{n} p_{2 n+4} u^{n}$, and so on. These functions have critical points at $u=u_{c}^{2}$ with the same exponents as those of (2). Our main objective is to obtain very accurate estimates for the connective constant $\mu$ and the critical exponents $\alpha$ and $\nu$. In particular we are keen to test a conjecture [3] for the exact value of the connective constant and confirm to a very high degree of precision the exact values of the exponents.

Once the exact values of the exponents have been confirmed we will turn our attention to the "fine structure" of the asymptotic form of the coefficients. In particular we are interested in obtaining accurate estimates for the amplitudes $B, D$ and $E^{(k)}$. We do this by fitting the coefficients to the assumed form (11). In this case our main aim is to test the validity of the predictions for the amplitude combinations mentioned in the Introduction.

\subsection{The polygon generating function}

In Table 3 we have listed estimates for the critical point $u_{c}^{2}$ and exponent $2-\alpha$ of the series for the square lattice SAP generating function. The estimates were obtained by averaging values obtained from second and third order differential approximants. For each order $L$ of the inhomogeneous polynomial we averaged over those approximants to the series which used at least the first 45 terms of the series (that is, polygons of perimeter at least 90). The error quoted for these estimates reflects the spread (basically one standard deviation) among the approximants. Note that these error bounds should not be viewed as a measure of the true error as they cannot include possible systematic sources of error. Based on these estimates we conclude that $u_{c}^{2}=0.14368062925(5)$ and $\alpha=0.5000001(2)$. This analysis adds strongly to the already very convincing evidence that the critical exponent $\alpha=1 / 2$ exactly.

Table 3: Estimates for the critical point $u_{c}^{2}$ and exponent $2-\alpha$ obtained from second and third order differential approximants to the series for square lattice polygon generating function. $L$ is the order of the inhomogeneous polynomial.

\begin{tabular}{lcccc}
\hline \hline$L$ & \multicolumn{2}{c}{ Second order DA } & \multicolumn{2}{c}{ Third order DA } \\
\hline & $u_{c}^{2}$ & $2-\alpha$ & $u_{c}^{2}$ & $2-\alpha$ \\
\hline 0 & $0.143680629242(28)$ & $1.500000116(94)$ & $0.143680629246(22)$ & $1.500000105(73)$ \\
2 & $0.143680629245(15)$ & $1.500000111(63)$ & $0.143680629247(21)$ & $1.500000097(81)$ \\
4 & $0.143680629246(16)$ & $1.500000107(62)$ & $0.143680629251(22)$ & $1.500000080(99)$ \\
6 & $0.143680629250(17)$ & $1.500000094(65)$ & $0.143680629244(22)$ & $1.500000109(72)$ \\
8 & $0.143680629249(22)$ & $1.500000094(72)$ & $0.143680629249(28)$ & $1.50000009(14)$ \\
10 & $0.143680629248(19)$ & $1.500000095(66)$ & $0.143680629252(28)$ & $1.50000006(15)$ \\
12 & $0.143680629246(21)$ & $1.500000105(70)$ & $0.143680629247(18)$ & $1.500000100(70)$ \\
14 & $0.143680629242(20)$ & $1.500000116(66)$ & $0.143680629245(26)$ & $1.500000099(99)$ \\
16 & $0.143680629252(18)$ & $1.500000086(63)$ & $0.143680629247(25)$ & $1.500000097(94)$ \\
18 & $0.143680629254(15)$ & $1.500000076(65)$ & $0.143680629247(22)$ & $1.500000098(81)$ \\
20 & $0.143680629238(26)$ & $1.500000122(74)$ & $0.143680629242(23)$ & $1.500000113(87)$ \\
\hline \hline
\end{tabular}

If we take the conjecture $\alpha=1 / 2$ to be true we can obtain a refined estimate for the critical point $u_{c}^{2}$ enabling us to check whether or not the estimates for $u_{c}^{2}$ still agree with 

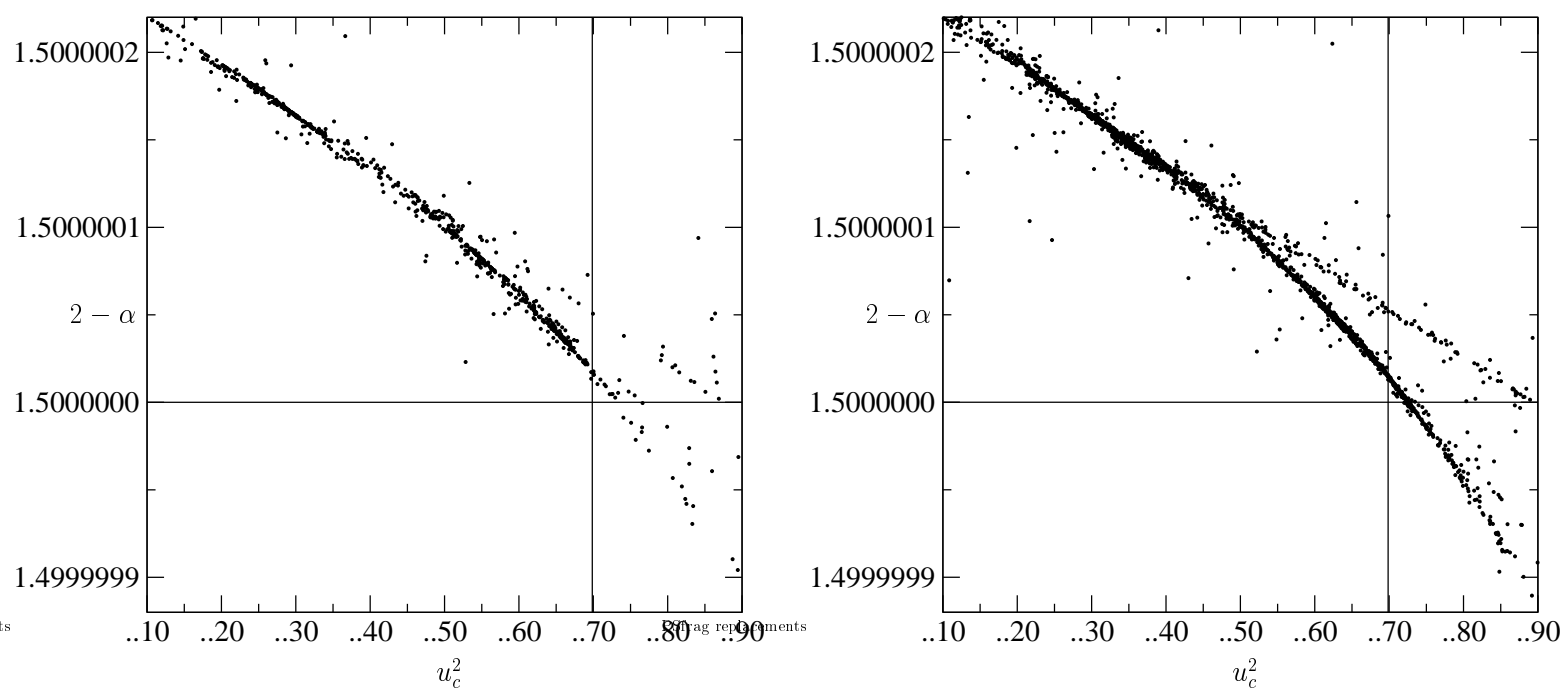

Figure 3: Estimates for the critical exponent $2-\alpha$ vs. estimates for the critical point $u_{c}^{2}$ of the square lattice polygon generating function. Each tick label along the $x$-axis is preceded by the value 0.1436806292 . The straight lines correspond to $2-\alpha=3 / 2$ and $u_{c}^{2}=0.1436806292698685 \ldots$

the root of the polynomial. In Fig. 3 we have plotted estimates for the critical exponent $2-\alpha$ against estimates for the critical point $u_{c}^{2}$. Each dot in the left (right) panel of this figure represents a pair of estimates obtained from a second (third) order inhomogeneous differential approximant. The order of the inhomogeneous polynomial was varied from 0 to 10 . As can be seen the estimates for the critical exponent cross the line $2-\alpha=3 / 2$ at a value $u_{c}^{2} \simeq 0.143680629273$, which is slightly larger than the value obtained from the root of the polynomial suggested as possibly providing the exact value. So this is the first direct evidence that the conjecture could be wrong. Since the difference only occurs in the 12th significant digit we do not feel confident that the numerical evidence alone is sufficient to disprove the conjecture. It may well be the case that there are subtle systematic trends in the estimates, which preclude them from having converged to the true values of the parameters. However, as emphasized in 18 the other zero of the polynomial is at $u_{c}^{2}=-0.1557288 \ldots$, and as was the case in this previous analysis, we see no evidence of such a singularity, which casts serious doubt on the validity of the conjecture. Particularly since we are not aware of any arguments as to why we might not expect to see the singularity on the negative real axis from our series analysis. Taken together these two pieces of 'evidence' may well be sufficient disprove to the conjecture. Ultimately we will let the reader make their own judgment.

Based on this analysis we adopt the value $u_{c}^{2}=0.143680629273(3)$ and thus $\mu=$ 2.63815853031(3) as our final estimates.

\subsection{The radius of gyration and area-weighted moments}

Table 4 contains estimates for $u_{c}^{2}$ and the critical exponents of the generating functions (2) for the radius of gyration and first area-weighted moment. Suffice to say, the estimates of the exponents are in agreement with the conjectured exact value $\nu=3 / 4$. 
Table 4: Estimates for the critical point $u_{c}^{2}$ and exponents $-(\alpha+2 \nu)$ and $2-(\alpha+2 \nu)$ obtained from second (top half) and third (bottom half) order differential approximants to the series for the radius of gyration and first area-weighted moment of square lattice SAP

\begin{tabular}{lllll}
\hline \hline Series: & \multicolumn{2}{c}{$\mathcal{R}_{g}^{2}(u)$} & \multicolumn{2}{c}{$\mathcal{P}^{(1)}(u)$} \\
\hline$L$ & \multicolumn{1}{c}{$u_{c}^{2}$} & \multicolumn{1}{c}{$2-\alpha$} & $u_{c}^{2}$ & \multicolumn{1}{c}{$2-\alpha$} \\
\hline 0 & $0.1436805865(92)$ & $-1.999681(36)$ & $0.1436806053(50)$ & $0.000122(15)$ \\
2 & $0.143680592(10)$ & $-1.999704(43)$ & $0.143680609(10)$ & $0.000143(60)$ \\
4 & $0.1436805889(82)$ & $-1.999689(35)$ & $0.143680609(11)$ & $0.000139(50)$ \\
6 & $0.143680583(23)$ & $-1.999676(82)$ & $0.143680604(12)$ & $0.00007(16)$ \\
8 & $0.143680588(10)$ & $-1.999680(55)$ & $0.143680608(10)$ & $0.00010(10)$ \\
10 & $0.143680591(12)$ & $-1.999703(59)$ & $0.143680616(22)$ & $-0.00011(79)$ \\
\hline 0 & $0.1436806081(85)$ & $-1.999822(53)$ & $0.143680607(12)$ & $0.00021(28)$ \\
2 & $0.143680605(13)$ & $-1.999803(79)$ & $0.143680616(11)$ & $0.00011(12)$ \\
4 & $0.1436806074(92)$ & $-1.999812(61)$ & $0.1436806143(73)$ & $0.000108(42)$ \\
6 & $0.143680606(11)$ & $-1.999817(71)$ & $0.1436806166(64)$ & $0.000095(26)$ \\
8 & $0.1436806057(93)$ & $-1.999809(53)$ & $0.1436806148(45)$ & $0.000083(40)$ \\
10 & $0.143680606(11)$ & $-1.999817(61)$ & $0.1436806154(55)$ & $0.000099(21)$ \\
\hline \hline
\end{tabular}

\subsection{The amplitudes}

The asymptotic form of the coefficients $p_{n}$ of the polygon generating function has been studied in detail previously [4, 18]. As argued in [4] there is no sign of non-analytic corrections-to-scaling exponents to the polygon generating function and one therefore finds that

$$
p_{n}=\mu^{n} n^{-5 / 2} \sum_{i \geq 0} a_{i} / n^{i}
$$

This form was confirmed with great accuracy in [18. Estimates for the leading amplitude $B=a_{0}$ can thus be obtained by fitting $p_{n}$ to the form given in equation (6) using increasing values of $k$. As in [16] we find it useful to check the behaviour of the estimates by plotting the results for the leading amplitude vs. $1 / n$ (see Fig. 4), where $p_{n}$ is the last term used in the fitting, and $n$ is varied from 110 down to 50. We again notice that as more and more correction terms are added to the fits the estimates exhibits less curvature and that the slope become less steep. This is very strong evidence that (6) indeed is the correct asymptotic form of $p_{n}$. We estimate that $B=0.56230130(2)$.

The asymptotic form of the coefficients $r_{n}$ in the generating function for the radius of gyration was studied in [16. When fitting to a form similar to equation (6), assuming that there are only analytic corrections-to-scaling, we found that the amplitudes of higher order terms are very large and that the leading amplitude converge rather slowly. This indicates that this asymptotic form is incorrect. We found that the coefficients fit better if we assume a leading non-analytic correction-to-scaling exponent $\Delta=3 / 2$. This result confirms the prediction of Nienhuis [25]. Note, that since the polygon generating function exponent $2-\alpha=3 / 2$ a correction-to-scaling exponent $\Delta=3 / 2$ is perfectly consistent 


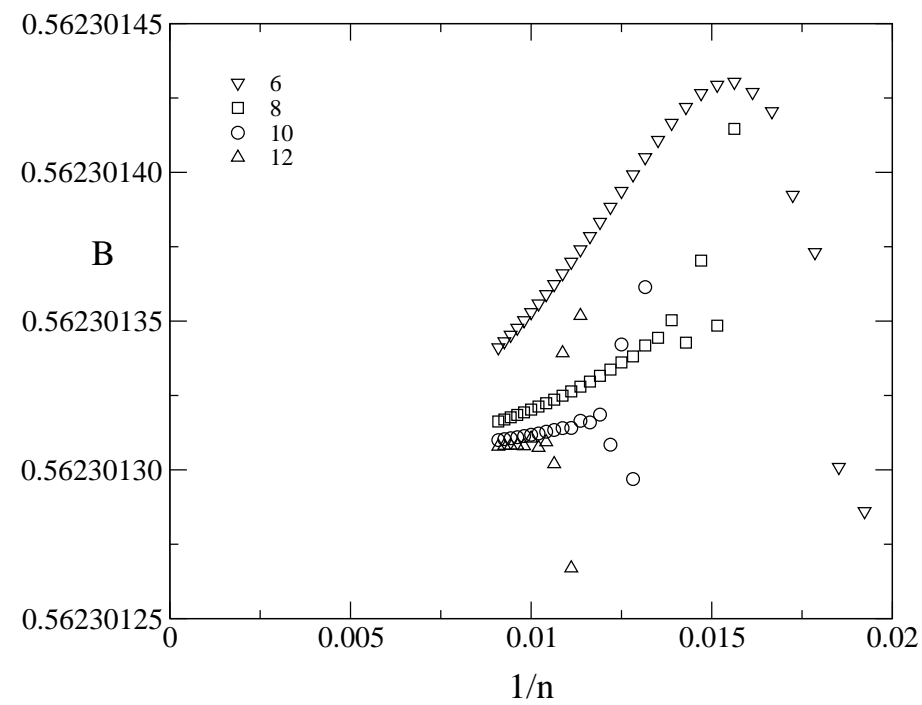

Figure 4: Estimates for the amplitude B vs. 1/n. Each data set is obtained by fitting $p_{n}$ to the form (6) using from 6 to 12 correction terms.
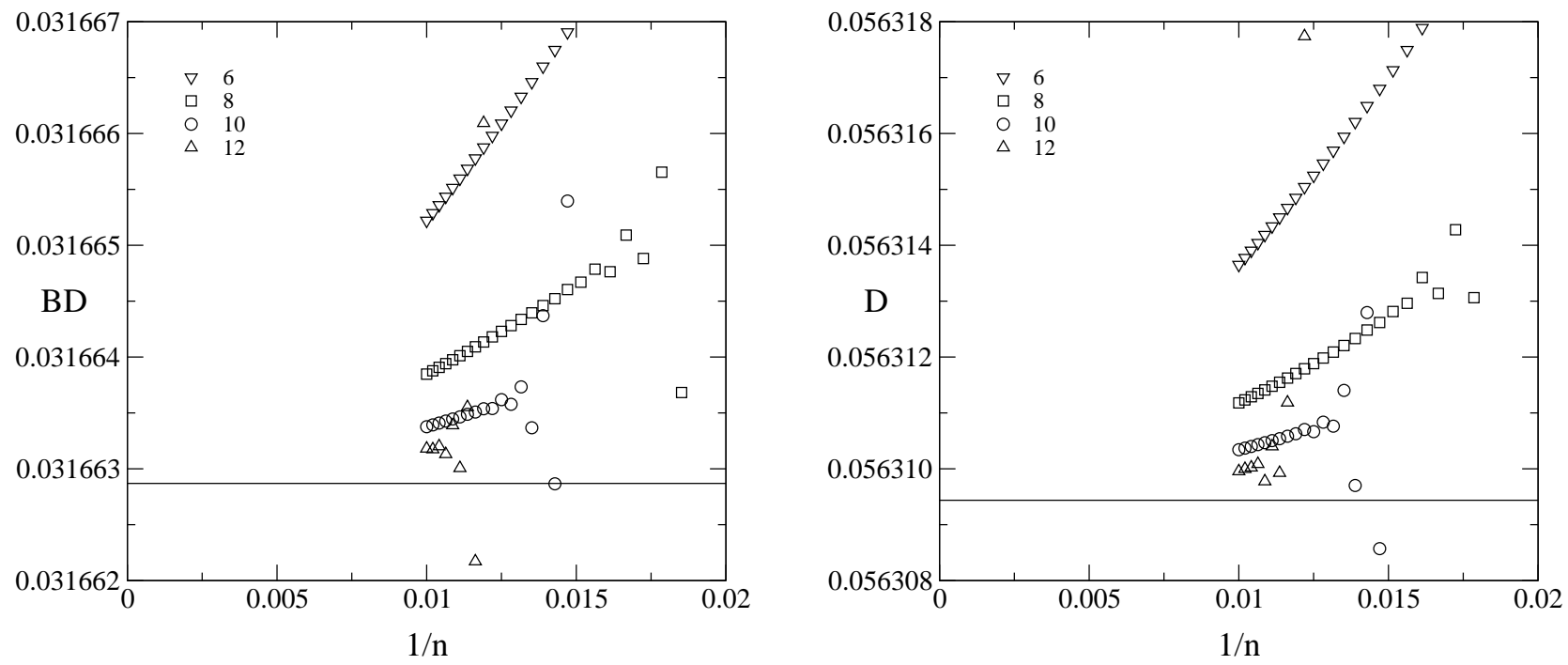

Figure 5: Estimates for the amplitudes BD and D vs. 1/n. Each data set is obtained by fitting $r_{n}$ to the form (17) and $r_{n} / p_{n}$ to the form (86) while using from 6 to 12 correction terms. 
Table 5: Exact values and estimates from square lattice polygons for the universal amplitude combinations.

\begin{tabular}{lll}
\hline \hline Amplitude & Exact value & Estimate \\
\hline$E^{(1)}$ & $0.795774715 \ldots \times 10^{-1}$ & $0.795773(2) \times 10^{-1}$ \\
$E^{(2)} B$ & $0.335953483 \ldots \times 10^{-2}$ & $0.335952(2) \times 10^{-2}$ \\
$E^{(2)} B^{2}$ & $0.100253732 \ldots \times 10^{-3}$ & $0.100253(1) \times 10^{-3}$ \\
$E^{(2)} B^{3}$ & $0.237553411 \ldots \times 10^{-5}$ & $0.237552(2) \times 10^{-5}$ \\
$E^{(2)} B^{4}$ & $0.475738345 \ldots \times 10^{-7}$ & $0.475736(3) \times 10^{-7}$ \\
$E^{(2)} B^{5}$ & $0.836630215 \ldots \times 10^{-9}$ & $0.836624(5) \times 10^{-9}$ \\
$E^{(2)} B^{6}$ & $0.132514776 \ldots \times 10^{-10}$ & $0.132514(2) \times 10^{-10}$ \\
$E^{(2)} B^{7}$ & $0.192419637 \ldots \times 10^{-12}$ & $0.192418(2) \times 10^{-12}$ \\
$E^{(2)} B^{8}$ & $0.259465635 \ldots \times 10^{-14}$ & $0.259464(2) \times 10^{-14}$ \\
$E^{(2)} B^{9}$ & $0.328063262 \ldots \times 10^{-16}$ & $0.328062(4) \times 10^{-16}$ \\
\hline \hline
\end{tabular}

with the asymptotic form (6). Because $2-\alpha+\Delta$ is an integer the non-analytic correction term becomes part of the analytic background term [4]. We thus proposed the following asymptotic form:

$$
r_{n}=\mu^{n} n\left[B D+\sum_{i \geq 0} a_{i} / n^{i / 2}\right] .
$$

Alternative we could fit to the form

$$
r_{n} / p_{n}=n^{7 / 2}\left[D+n^{5 / 2} \sum_{i \geq 0} a_{i} / n^{i / 2}\right] .
$$

In figure 5 we show the leading amplitudes resulting from such fits while using from 1 to 10 terms in these expansions. Also shown in these figures (solid lines) are the predicted exact value of $B D$, given in equation 4 , and the prediction for $D$ using the estimate for $B$ obtained above. As can be seen the leading amplitudes clearly converge towards their expected values and from these plots we can conclude that the prediction for $B D$ has been confirmed to at least 6 digit accuracy. Assuming that equation (4) is exact and using the very accurate estimate for $B$ we find that $D=0.056309437(2)$.

Next we test the predictions [27] for the amplitudes of the area-weighted moments. We fit the the coefficients to the assumed form

$$
a_{n}^{(k)} \approx \mu^{n} n^{(\alpha+2 k \nu)-1}\left[E^{(k)}+\sum_{i \geq 0} a_{i} / n^{1+i / 2}\right] .
$$

We obtain several data sets by varying the number of terms using in this formula from 8 to 12. To obtain the final estimates we do a simple linear regression on the data for the amplitudes as a function of $1 / n$ extrapolating to $1 / n \rightarrow 0$. We estimate the error on the amplitude estimate from the spread among the different data sets. In this way, we obtain the results for the leading amplitudes listed in Table 5 .

It is clear that the results for the first 10 area weighted moments are in excellent agreement with the numerical estimates. On this basis we conclude that the conjectured scaling function and derived exact amplitude combinations [27] are correct. 


\section{Conclusion}

We have presented an improved and parallel algorithm for the enumeration of self-avoiding polygons on the square lattice. This algorithm has enabled us to obtain polygons up to perimeter length 110 and their radius of gyration and area-weighted moments up to perimeter 100. Our extended series enables us to give an extremely precise estimate of the connective constant $\mu=2.63815853031(3)$. This estimate provides the first direct evidence that an earlier conjecture for the exact value of $\mu$ could be incorrect. We confirmed to a very high degree of accuracy the predicted exponent values $\alpha=1 / 2$ and $\nu=3 / 4$. We also obtained very accurate estimates for the leading amplitude $B=0.56230130(2)$ of the asymptotic expansion of $p_{n}$, and confirmed the correctness of theoretical predictions for the values of the amplitude combinations $B D$ and $E^{(k)} B^{k-1}$.

\section{E-mail or WWW retrieval of series}

The series for the generating functions studied in this paper can be obtained via e-mail by sending a request to I.Jensen@ms.unimelb.edu.au or via the world wide web on the URL http://www.ms.unimelb.edu.au/ iwan/ by following the instructions.

\section{Acknowledgments}

The calculations presented in this paper would not have been possible without a generous grant of computer time on the server cluster of the Australian Partnership for Advanced Computing (APAC). We also used the computational resources of the Victorian Partnership for Advanced Computing (VPAC). We gratefully acknowledge financial support from the Australian Research Council.

\section{References}

[1] Cardy J L 1994 Mean area of self-avoiding loops Phys. Rev. Lett. 72 1580-1583

[2] Cardy J L and Guttmann A J 1993 Universal amplitude combinations for selfavoiding walks, polygons and trails J. Phys. A: Math. Gen. 26 2485-2494

[3] Conway A R, Enting I G and Guttmann A J 1993 Algebraic techniques for enumerating self-avoiding walks on the square lattice J. Phys. A: Math. Gen. 26 1519-1534

[4] Conway A R and Guttmann A J 1996 Square lattice self-avoiding walks and corrections to scaling Phys. Rev. Lett. 77 5284-5287

[5] Delest M P and Viennot G 1984 Algebraic languages and polyominoes enumeration Theor. Comput. Scie. 34 169-206

[6] Enting I G 1980 Generating functions for enumerating self-avoiding rings on the square lattice J. Phys. A: Math. Gen. 13 3713-3722

[7] Enting I G and Guttmann A J 1985 Self-avoiding polygons on the square, L and Manhattan lattices J. Phys. A: Math. Gen. 18 1007-1017 
[8] Enting I G and Guttmann A J 1990 On the area of square lattice polygons J. Stat. Phys. 58 475-484

[9] Enting I G and Guttmann A J 1992 Self-avoiding rings on the triangular lattice J. Phys. A: Math. Gen. 25 2791-2807

[10] Fisher M E 1989 Fractal and nonfractal shapes in two-dimensional vesicles Physica D 38 112-118

[11] Fisher M E, Guttmann A J and Whittington S G 1991 Two-dimensional lattice vesicles and polygons J. Phys. A: Math. Gen. 24 3095-3106

[12] Guttmann A J 1989 Asymptotic analysis of power-series expansions in Phase Transitions and Critical Phenomena (eds. C Domb and J L Lebowitz) (New York: Academic) vol. 13 pp. 1-234

[13] Guttmann A J and Enting I G 1988 The size and number of rings on the square lattice J. Phys. A: Math. Gen. 21 L165-172

[14] Hiley B J and Sykes M F 1961 Probability of initial ring close in the restricted random-walk model of a macromolecule J. Chem. Phys. 34 1531-1537

[15] Hughes B D 1995 Random Walks and Random Environments, Vol I Random Walks (Oxford: Clarendon)

[16] Jensen I 2000 Size and area of square lattice polygons J. Phys. A: Math. Gen. 33 3533-3543

[17] Jensen I and Guttmann A J 1998 Self-avoiding walks, neighbour-avoiding walks and trails on semiregular lattices J. Phys. A: Math. Gen. 30 8137-8145

[18] Jensen I and Guttmann A J 1999 Self-avoiding polygons on the square lattice J. Phys. A: Math. Gen. 32 4867-4876

[19] Knuth D E 1969 Seminumerical Algorithms. The Art of Computer Programming, Vol 2. (Reading, Mass: Addison Wesley)

[20] Knuth D E 2001 Polynum and Polyslave the program is available from Knuth's Home-page at http://Sunburn.Stanford.EDU ${ }^{\sim}$ knuth/programs.html\#polyominoes

[21] Leibler S, Singh R R P and Fisher M E 1987 Thermodynamic behavior of twodimensional vesicles Phys. Rev. Lett. 59 1989-1992

[22] Lin K Y 2000 Universal amplitude combinations for self-avoiding walks and polygons on the honeycomb lattice Physica A 275 197-206

[23] Lin K Y and Kao Y M 1999 Universal amplitude combinations for self-avoiding walks and polygons on directed lattices J. Phys. A: Math. Gen. 32 6927-6938

[24] Lin K Y and Lue S J 1999 Universal amplitude combinations for self-avoiding polygons on the kagome lattice Physica A 270 453-461 
[25] Nienhuis B 1982 Exact critical point and critical exponents of $\mathrm{O}(n)$ models in two dimensions Phys. Rev. Lett. 49 1062-1065

[26] Privman V and Rudnick J 1985 Size of rings in two dimensions J. Phys. A: Math. Gen. 18 L789-L793

[27] Richard C, Guttmann A J and Jensen I 2001 Scaling function and universal amplitude combinations for self-avoiding polygons J. Phys. A: Math. Gen. 34 L495-L501 